\title{
On the Error Probability of Interference Exploitation Precoding with Power Allocation
}

\author{
Abdelhamid Salem and Christos Masouros \\ Department of Electronic and Electrical Engineering, \\ University College London, London, UK \\ emails: \{a.salem, c.masouros\}@ucl.ac.uk
}

\begin{abstract}
In this paper, we analyze the performance of constructive interference (CI) precoding in downlink multi-user multiple-input single-output (MU-MISO) systems with phaseshift-keying (PSK) signals. A new closed-form expression is derived for the moment generating function (MGF) of the received signal-to-noise-ratio (SNR). Then, the MGF is used to calculate the average symbol error probability (SEP) for the CI technique. In light of this, new exact analytical expression and very accurate asymptotic expression for the average SEP are presented. Based on the new SEP expressions, a power allocation scheme to minimize the sum SEPs (Min-Sum) is investigated, and analytical expression of the power allocation factors is derived. The numerical results show that, the CI precoding yields superior performance over conventional interference suppression precoding techniques in terms of SEP. Furthermore, the Min-Sum power allocation scheme provides additional up to $10 \mathrm{~dB}$ gains in the transmit SNR compared to equal power allocation technique.
\end{abstract}

Index Terms-Interference exploitation, multiple users MIMO, PSK.

\section{INTRODUCTION}

Multi-user multiple-input single-output (MU-MISO) communication systems play important roles in achieving high reliability, and spectral-efficiency [1], [2]. However, the multiuser interference in MU-MISO systems is the main cause of performance degradation in practical implementations. Consequently, a large number of researches have been conducted on canceling the interference in MU-MISO systems [2], [3]. For instance, dirty-paper coding (DPC) technique has been introduced to eliminate the interference in applications when the channel state information (CSI) is perfectly known at the base station (BS) [4]. Nevertheless, it is difficult to implement DPC in practical applications, because of its very high complexity. Consequently, low-complexity linear precoding schemes, such as zero-forcing (ZF), have received significant attention in recent years [5], [6]. Furthermore, precoding design schemes that based on optimization have also widely investigated [7], [8]. For instance, signal to interference-plus-noise ratio (SINR) balancing technique is a precoding scheme that aims to optimize the minimum SINR subject to power constraints [7], [8]. However, all the aforementioned precoding techniques have ignored the fact that the multi-user interference can be beneficial to the received symbols, and thus it can be exploited to increase the received power of the useful signals. Based on this fact, constructive interference (CI) exploitation technique has been proposed to enhance the performance of
MU-MISO systems [8]-[10]. The interference is considered to be constructive if it pushes the received symbols deeper in the constructive region of the desired symbol. Therefore, with the knowledge of both the users' channels and users' data symbols, the precoder can be designed to make all the multi-user interference constructive to the received symbols. The concept of interference exploitation technique has been extensively investigated over the past few years. This concept was introduced in [9], where the CI precoding was proposed for down-link MIMO systems. The results in [9] showed that the CI precoding can improve the received SINR without increasing the transmission power. In [10] a low-complexity vector precoding scheme for $\mathrm{CI}$ in down-link MU-MISO system was proposed. The authors in [8] presented new beamforming techniques for MU-MISO systems in order to minimize the transmission power by using the concept of the CI exploitation technique. Recently, closed-form expression for CI precoding in MU-MISO systems under PSK signaling has been derived in [7]. Based on this closed-form expression, the CI exploitation approach has been analytically investigated in [11]-[13].

Accordingly this paper analyzes the performance of CI precoding with $M$-PSK signals in MU-MISO systems. Firstly, we derive exact analytical expression of the moment-generating function (MGF) of the received SNR. Based on this, we calculate the average symbol error probability (SEP) for CI precoding with $M$-PSK signals. In this context, exact analytical expression for the SEP is derived. Additionally and to provide more insights, new accurate approximation for the SEP is also presented. Building on the new SEP expressions, a power allocation scheme to minimize the sumSEPs (Min-Sum) is considered, and closed form expression of the power allocation factors is derived. The results in this paper demonstrate that, for a given SEP value the CI precoding provides up to $16 \mathrm{~dB}$ gain in the transmit SNR compared to the traditional interference suppression, ZF, precoding scheme. Furthermore, a significant improvement in the average SEP can be attained using the Min-Sum power allocation scheme compared to the uniform power allocation scheme.

\section{System MOdEL}

We consider a down-link MU-MISO system, where $N$ antenna BS transmits signals to $K$ single antenna users. The down-link $K \times N$ channel matrix between the BS and the users 
is denoted by $\mathbf{G}$, which is modeled as $\mathbf{G}=\mathbf{D}^{1 / 2} \tilde{\mathbf{G}}$, where the $K \times N$ matrix $\tilde{\mathbf{G}}$ represents the small-scale fading coefficients from the BS to the users which are assumed to be independent, circularly symmetric complex Gaussian random variables with zero mean and unit variance, and $\mathbf{D}$ is a $K \times K$ diagonal matrix models the path-loss where $[\mathbf{D}]_{k k}=\varpi_{k}=d_{k}^{-m}, d_{k}$ is the distance from the BS to the $k^{t h}$ user and $m$ is the pathloss exponent. Therefore, the received signal at user $k$ can be written as

$$
y_{k}=\mathbf{g}_{k} \mathbf{W} \mathbf{x}+n_{k}
$$

where $\mathbf{x}$ is the PSK-modulated signal vector, $\mathbf{W}$ is the precoding matrix, $\mathbf{g}_{k}$ is the channel vector between the BS and the $k^{t h}$ user, and $n_{k}$ is the additive wight Gaussian noise (AWGN) at the $k^{t h}$ user, $n_{k} \sim \mathcal{C N}\left(0, \sigma_{k}^{2}\right)$. The CI precoding matrix with PSK signaling is given by [7], [11], [12]

$$
\mathbf{W}=\frac{1}{K} \beta \mathbf{G}^{H}\left(\mathbf{G G}^{H}\right)^{-1} \operatorname{diag}\left\{\mathbf{V}^{-1} \mathbf{u}\right\} \mathbf{x} \mathbf{x}^{H},
$$

where $\beta=\sqrt{P_{p}} \beta_{p}, P_{p}$ is the total transmit power and $\beta_{P}$ is the power scaling factor, $\beta_{p}=\sqrt{\frac{1}{\mathbf{u}^{H} \mathbf{V}^{-1} \mathbf{u}}}$, while $\mathbf{V}=\operatorname{diag}\left(\mathbf{x}^{H}\right)\left(\mathbf{G G}^{H}\right)^{-1} \operatorname{diag}(\mathbf{x})$ and $\mathbf{1}^{H} \mathbf{u}=1$. In the $\mathrm{CI}$ technique the resulting interference contributes to the desired signal power. Consequently, the received SNR at user $k$ using CI precoding can be expressed as [8], [14]

$$
\gamma_{k}=\frac{\left|\boldsymbol{g}_{k} \mathbf{W} \mathbf{x}\right|^{2}}{\sigma_{k}^{2}}
$$

Next, the MGF of the received SNR and the performance of CI precoding in terms of the average SEP are considered.

\section{MGF DERIVATION}

In this section, we derive the MGF of the received SNR in (3). To start with, by substituting (2) into (3), the received SNR at user $k$ can be expressed as

$$
\gamma_{k}=\frac{\left|\boldsymbol{g}_{k} \frac{\sqrt{P_{p}} \beta_{p}}{K} \mathbf{G}^{H}\left(\mathbf{G G}^{H}\right)^{-1} \operatorname{diag}\left\{\mathbf{V}^{-1} \mathbf{u}\right\} \mathbf{x}\right|^{2}}{\sigma_{k}^{2}}
$$

For mathematical tractability, the scaling factor $\beta_{p}$ is designed to constrain the long-term total power transmission, and thus it can be presented as $\beta_{p}=\frac{1}{\sqrt{\mathcal{E}\left\{\mathbf{u}^{H} \mathbf{V}^{-1} \mathbf{u}\right\}}}$ [3], [7]. Given that, the matrix $\left(\mathbf{G G}{ }^{H}\right)$ has Wishart distribution, we can find that, $\beta_{p}=\frac{1}{\sqrt{\mathbf{u}^{H} \operatorname{diag}\left(\mathbf{x}^{H}\right)^{-1} N \mathbf{\Sigma}(\operatorname{diag}(\mathbf{x}))^{-1} \mathbf{u}}}$, where $\Sigma=\mathrm{D}$ [15]. The last formula in (4) can be written also as

$$
\gamma_{k}=\frac{\left|\frac{\sqrt{P_{p}} \beta_{p}}{K} \mathbf{b} \Sigma \mathbf{u} \frac{\mathbf{b} \mathbf{A} \mathbf{u}}{\mathbf{b} \Sigma \mathbf{u}} x_{k}\right|^{2}}{\sigma_{k}^{2}}=\alpha_{k}|h|^{2}
$$

where $\mathbf{b}=\mathbf{a}_{k}, \mathbf{a}_{k}$ is a $1 \times K$ vector the elements of this vector are zeros and the $k^{\text {th }}$ element of this vector is one, and $\mathbf{A}=\mathbf{V}^{-1}, \alpha_{k}=\frac{\left|\frac{\sqrt{P_{p}} \beta_{p}}{\kappa} \mathbf{b} \Sigma \mathbf{u}\right|^{2}}{\sigma_{k}^{2}}$ and $h=\frac{\mathbf{b} \mathbf{A u}}{\mathbf{b} \Sigma \mathbf{u}}$. The distribution of $h=\frac{\mathbf{b} \mathbf{A u}}{\mathbf{b} \Sigma \mathbf{u}}$ can be approximated to Gamma distribution [15]. Therefore, the MGF of the received SNR can be derived as

$$
\begin{gathered}
\mathcal{M}_{\gamma_{k}}(z)=\int_{0}^{\infty} e^{-z \gamma} f_{\gamma_{k}}(\gamma) d \gamma \\
\mathcal{M}_{\gamma}(z)=\int_{0}^{\infty} e^{-z \alpha_{k}|h|^{2}}\left(\frac{h^{N-1} e^{-h}}{(N-1) !}\right) d h
\end{gathered}
$$

This expression can be simplified using Gaussian Quadrature rule as

$$
\mathcal{M}_{\gamma}(z)=\sum_{i=1}^{n} \mathrm{H}_{i} e^{-z P_{p} \zeta_{k}\left|h_{i}\right|^{2}}\left(\frac{h_{i}^{N-1}}{(N-1) !}\right)
$$

where $\zeta_{k}=\frac{\left|\frac{\beta_{p}}{K} \mathbf{b} \Sigma \mathbf{u}\right|^{2}}{\sigma_{k}^{2}}, \mathrm{H}_{i}$ and $h_{i}$ are the $i^{\text {th }}$ zero and the weighting factor of the Laguerre polynomials, respectively [16].

\section{Average Symbol ERror Probability (SEP)}

The average SEP with $M$-PSK, can be written as [17, (5.67)]

$$
P_{e, k}=\frac{1}{\pi} \int_{0}^{\frac{\pi(M-1)}{M}} \mathcal{M}_{\gamma}\left(-\frac{\sin ^{2}\left(\frac{\pi}{M}\right)}{\sin ^{2} \Phi}\right) d \Phi
$$

In the following, the exact and approximate formulas of the average SEP are derived.

\section{A. Exact SEP}

For simplicity, we can write (9) as

$$
P_{e, k}=\frac{1}{\pi} \int_{0}^{\Theta} \mathcal{M}_{\gamma}(z) d \theta
$$

where $\Theta=\frac{\pi(M-1)}{M}$ and $z=-\frac{\sin ^{2}\left(\frac{\pi}{M}\right)}{\sin ^{2} \Phi}$. By Substituting (8) into (10), we can get

$$
P_{e, k}=\frac{1}{\pi} \int_{0}^{\Theta} \sum_{i=1}^{n} \mathrm{H}_{i} e^{-\left(\frac{\sin ^{2}\left(\frac{\pi}{M}\right)}{\sin ^{2} \Phi}\right) P_{p} \zeta_{k}\left|h_{i}\right|^{2}}\left(\frac{h_{i}^{N-1}}{(N-1) !}\right) d \Phi
$$

and

$$
P_{e, k}=\frac{1}{\pi} \sum_{i=1}^{n} \frac{\mathrm{H}_{i} h_{i}^{N-1}}{(N-1) !} \int_{0}^{\Theta} e^{-\left(\frac{\sin ^{2}\left(\frac{\pi}{M}\right)}{\sin ^{2} \Phi}\right) P_{p} \zeta_{k}\left|h_{i}\right|^{2}} d \Phi
$$

As we can see from (12), the exact SEP expression is obtained with single integration which can be evaluated efficiently using numerical integration methods. 


\section{B. Approximate SEP}

Here we provide very accurate approximation of the average SEP. Firstly, (10) can be written as

$$
\begin{gathered}
P_{e, k}=\mathcal{E}\left[\frac{1}{\pi} \int_{0}^{\frac{\pi}{2}} e^{\left(-\frac{\sin ^{2}\left(\frac{\pi}{M}\right)}{\sin ^{2} \theta}\right)} d \theta\right. \\
\left.+\frac{1}{\pi} \int_{\frac{\pi}{2}}^{\Theta} e^{\left(-\frac{\sin ^{2}\left(\frac{\pi}{M}\right)}{\sin ^{2} \theta}\right)} d \theta\right]
\end{gathered}
$$

The first and the second terms in (13) can be efficiently approximated by [18], [19]

$$
\frac{1}{\pi} \int_{0}^{\frac{\pi}{2}} e^{\left(-\frac{\sin ^{2}\left(\frac{\pi}{M}\right)}{\sin ^{2} \theta}\right)} d \theta \approx \frac{1}{12} e^{\left(-\sin ^{2}\left(\frac{\pi}{M}\right)\right)}+\frac{1}{4} e^{\left(-\frac{4 \sin ^{2}\left(\frac{\pi}{M}\right)}{3}\right)}
$$

$$
\begin{aligned}
& \frac{1}{\pi} \int_{\frac{\pi}{2}}^{\Theta} e^{\left(-\frac{\sin ^{2}\left(\frac{\pi}{M}\right)}{\sin ^{2} \theta}\right)} d \theta \approx \\
& \frac{1}{2 \pi}\left(e^{\left(-\sin ^{2}\left(\frac{\pi}{M}\right)\right)}+\frac{1}{4} e^{\left(-\frac{\sin ^{2}\left(\frac{\pi}{M}\right)}{\sin ^{2} \Theta}\right)}\right)\left(\Theta-\frac{\pi}{2}\right)
\end{aligned}
$$

Now substituting (14) and (15) into (13), the approximate expression of the average SEP can be obtained as [18], [19]

$$
\begin{aligned}
P_{e, k} & =\mathcal{E}\left[\frac{1}{12} e^{\left(-\sin ^{2}\left(\frac{\pi}{M}\right)\right)}+\frac{1}{4} e^{\left(-\frac{4 \sin ^{2}\left(\frac{\pi}{M}\right)}{3}\right)}\right. \\
& \left.+\frac{1}{2 \pi}\left(e^{\left(-\sin ^{2}\left(\frac{\pi}{M}\right)\right)}+\frac{1}{4} e^{\left(-\frac{\sin ^{2}\left(\frac{\pi}{M}\right)}{\sin ^{2} \Theta}\right)}\right)\left(\Theta-\frac{\pi}{2}\right)\right]
\end{aligned}
$$

which can be expressed as

$$
\begin{array}{r}
P_{e, k}=\left(\frac{\Theta}{2 \pi}-\frac{1}{6}\right) \mathcal{M}_{\gamma}\left(\sin ^{2}\left(\frac{\pi}{M}\right)\right)+\frac{1}{4} \mathcal{M}_{\gamma}\left(\frac{4 \sin ^{2}\left(\frac{\pi}{M}\right)}{3}\right) \\
+\left(\frac{\Theta}{2 \pi}-\frac{1}{4}\right) \mathcal{M}_{\gamma}\left(\frac{\sin ^{2}\left(\frac{\pi}{M}\right)}{\sin ^{2} \Theta}\right)
\end{array}
$$

Finally, substituting (8) into (17), the approximate expression of the average SEP can be written as in (18), shown at the top of the next page.

The numerical results in Section (VI) will show that, the approximate SEP expression in (18) is very accurate.

\section{Error Minimization Through Power Allocation}

The main aim of this part is to use the analytical expressions derived in the previous sections to minimize the sum-SEPs of the CI precoding with non equal power allocation (EPA) subject to total power constraint. Therefore, the optimization problem of this power-allocation scheme can be formulated as

$$
\begin{gathered}
\min _{\boldsymbol{a}} \mathbf{1}_{K}^{T} \mathfrak{p} \\
\text { S.t }: \sum_{k=1}^{K} a_{k}=1, a_{k} \geq 0
\end{gathered}
$$

where $\mathfrak{p}=\left[P_{e, 1}, \ldots, P_{e, k}, \ldots, P_{e, K}\right]^{T}$ is the users' SEP vector, and $\boldsymbol{a}=\left[a_{1}, \ldots, a_{k}, \ldots, a_{K}\right]$ is the relative power allocation vector. The optimization problem in (19) can also be written as

$$
\begin{gathered}
\min _{\boldsymbol{a}} \sum_{k=1}^{K} P_{e, k} \\
\text { S.t }: \sum_{k=1}^{K} a_{k}=1, a_{k} \geq 0
\end{gathered}
$$

Substituting (18) into (20), we can get

$$
\begin{gathered}
\min _{a_{k}} \sum_{k=1}^{K}\left\{c_{1}\left[\sum_{i=1}^{n} \vartheta_{i} e^{-z_{1} a_{k} P_{p} \zeta_{k}\left|h_{i}\right|^{2}}\right]\right. \\
\left.+c_{2}\left[\sum_{i=1}^{n} \vartheta_{i} e^{-z_{2} a_{k} P_{p} \zeta_{k}\left|h_{i}\right|^{2}}\right]+c_{3}\left[\sum_{i=1}^{n} \vartheta_{i} e^{-z_{3} a_{k} P_{p} \zeta_{k}\left|h_{i}\right|^{2}}\right]\right\} \\
\text { S.t }: \sum_{k=1}^{K} a_{k}=1, a_{k} \geq 0
\end{gathered}
$$

where $c_{1}=\frac{\left(\frac{(M-1)}{2 M}-\frac{1}{6}\right)}{(N-1) !}, c_{2}=\frac{1}{4(N-1) !}, c_{3}=\frac{\left(\frac{(M-1)}{2 M}-\frac{1}{4}\right)}{(N-1) !}$, $\vartheta_{i}=h_{i}^{N-1} \mathrm{H}_{i}, z_{1}=\sin ^{2}\left(\frac{\pi}{M}\right), z_{2}=\frac{4 \sin ^{2}\left(\frac{\pi}{M}\right)}{3}$ and $z_{3}=\frac{\sin ^{2}\left(\frac{\pi}{M}\right)}{\sin ^{2} \frac{\pi(M-1)}{M}}$. The function in (21) is convex in the parameters $a_{k}$ over the feasible set defined by linear power ratio constraints, $\frac{\partial^{2}}{\partial a_{k}^{2}} P_{e, k}>0$ for $a_{k}>0$. Consequently, the problem in (21) can be optimally solved using software tools. However, in order to gain some insights into the power allocation strategy, we consider numerical solution as follows. Following the definitions in [20], the Lagrangian of the optimization problem in (21) can be written as,

$$
\mathfrak{L}(\mathfrak{p}, \lambda)=\mathbf{1}_{K}^{T} \mathfrak{p}+\lambda\left(\sum_{k=1}^{K} a_{k}-1\right)
$$

where $\lambda$ is the Lagrange multiplier satisfying the power constraint. Therefore, the solution can be obtained from the conditions

$$
\frac{\partial}{\partial \lambda} \mathfrak{L}(\mathfrak{p}, \lambda)=\left(\sum_{k=1}^{K} a_{k}-1\right)=0
$$




$$
\begin{aligned}
P_{e, k}=\left(\frac{(M-1)}{2 M}-\frac{1}{6}\right) & \sum_{i=1}^{n} \mathrm{H}_{i} e^{-\left(\sin ^{2}\left(\frac{\pi}{M}\right)\right) P_{p} \zeta_{k}\left|h_{i}\right|^{2}}\left(\frac{h_{i}^{N-1}}{(N-1) !}\right)+\frac{1}{4} \sum_{i=1}^{n} \mathrm{H}_{i} e^{-\left(\frac{4 \sin ^{2}\left(\frac{\pi}{M}\right)}{3}\right) P_{p} \zeta_{k}\left|h_{i}\right|^{2}}\left(\frac{h_{i}^{N-1}}{(N-1) !}\right) \\
& +\left(\frac{(M-1)}{2 M}-\frac{1}{4}\right) \sum_{i=1}^{n} \mathrm{H}_{i} e^{-\left(\frac{\sin ^{2}\left(\frac{\pi}{M}\right)}{\sin ^{2} \frac{\pi(M-1)}{M}}\right) P_{p} \zeta_{k}\left|h_{i}\right|^{2}}\left(\frac{h_{i}^{N-1}}{(N-1) !}\right)
\end{aligned}
$$

$$
\frac{\partial}{\partial a_{k}} \mathfrak{L}(\mathfrak{p}, \lambda)=\lambda-\psi_{k}=0
$$

where $\quad \psi_{k} \quad=\quad c_{1}\left[\sum_{i=1}^{n} \omega_{i 1, k} \vartheta_{i} e^{-\omega_{i 1, k} a_{k}}\right]+$ $c_{2}\left[\sum_{i=1}^{n} \omega_{i 2, k} \vartheta_{i} e^{-a_{k} \omega_{i 2, k}}\right]+c_{3}\left[\sum_{i=1}^{n} \omega_{i 3, k} \vartheta_{i} e^{-a_{k} \omega_{i 3, k}}\right]$, $\omega_{i j, k}=z_{j} P_{p} \zeta_{k}\left|h_{i}\right|^{2}, j=1,2,3$. From (24), it can be noted that $\psi_{k}=\psi_{k-1}=\ldots=\psi_{1}$, thus,

$$
\begin{aligned}
& c_{1}\left[\sum_{i=1}^{n} \omega_{i 1, k} \vartheta_{i} e^{-\omega_{i 1, k} a_{k}}\right]+c_{2}\left[\sum_{i=1}^{n} \omega_{i 2, k} \vartheta_{i} e^{-a_{k} \omega_{i 2, k}}\right] \\
& +c_{3}\left[\sum_{i=1}^{n} \omega_{i 3, k} \vartheta_{i} e^{-a_{k} \omega_{i 3, k}}\right]=c_{1}\left[\sum_{i=1}^{n} \omega_{i 1,1} \vartheta_{i} e^{-\omega_{i 1,1} a_{1}}\right] \\
& +c_{2}\left[\sum_{i=1}^{n} \omega_{i 2,1} \vartheta_{i} e^{-a_{1} \omega_{i 2,1}}\right]+c_{3}\left[\sum_{i=1}^{n} \omega_{i 3,1} \vartheta_{i} e^{-a_{1} \omega_{i 3,1}}\right]
\end{aligned}
$$

Considering the first-order laguerre-polynomial, we can get

$$
\begin{aligned}
&\left(\frac{(M-1)}{2 M}-\right.\left.\frac{1}{6}\right) \zeta_{k} e^{-z_{1} P_{p} \zeta_{k}\left|h_{1}\right|^{2} a_{k}}+\frac{1}{3} \zeta_{k} e^{-a_{k} z_{2} P_{p} \zeta_{k}\left|h_{1}\right|^{2}} \\
&+\frac{\frac{(M-1)}{2 M}-\frac{1}{4}}{\sin ^{2} \frac{\pi(M-1)}{M}} \zeta_{k} e^{-a_{k} z_{3} P_{p} \zeta_{k}\left|h_{1}\right|^{2}}= \\
&\left(\frac{(M-1)}{2 M}-\frac{1}{6}\right) \zeta_{1} e^{-z_{1} P_{p} \zeta_{1}\left|h_{1}\right|^{2} a_{1}}+\frac{1}{3} \zeta_{1} e^{-a_{1} z_{2} P_{p} \zeta_{1}\left|h_{1}\right|^{2}} \\
&+\frac{\left(\frac{(M-1)}{2 M}-\frac{1}{4}\right)}{\sin ^{2} \frac{\pi(M-1)}{M}} \zeta_{1} e^{-a_{1} z_{3} P_{p} \zeta_{1}\left|h_{1}\right|^{2}}
\end{aligned}
$$

For a given $\zeta_{1}$ and $\zeta_{k}$, we can find

$$
\begin{aligned}
e^{-z_{1} P_{p} \zeta_{k}\left|h_{1}\right|^{2} a_{k}} & =\frac{\zeta_{1}}{\zeta_{k}} e^{-z_{1} P_{p} \zeta_{1}\left|h_{1}\right|^{2} a_{1}} \\
e^{-a_{k} z_{2} P_{p} \zeta_{k}\left|h_{1}\right|^{2}} & =\frac{\zeta_{1}}{\zeta_{k}} e^{-a_{1} z_{2} P_{p} \zeta_{1}\left|h_{1}\right|^{2}} \\
e^{-a_{k} z_{3} P_{p} \zeta_{k}\left|h_{1}\right|^{2}} & =\frac{\zeta_{1}}{\zeta_{k}} e^{-a_{1} z_{3} P_{p} \zeta_{1}\left|h_{1}\right|^{2}}
\end{aligned}
$$

which can also be written as

$$
a_{k}=\frac{\zeta_{1} a_{1}}{\zeta_{k}}-\frac{\ln \frac{\zeta_{1}}{\zeta_{k}}}{z_{1} P_{p} \zeta_{k}\left|h_{1}\right|^{2}}
$$

$$
\begin{aligned}
& a_{k}=\frac{\zeta_{1} a_{1}}{\zeta_{k}}-\frac{\ln \frac{\zeta_{1}}{\zeta_{k}}}{z_{2} P_{p} \zeta_{k}\left|h_{1}\right|^{2}} \\
& a_{k}=\frac{\zeta_{1} a_{1}}{\zeta_{k}}-\frac{\ln \frac{\zeta_{1}}{\zeta_{k}}}{z_{3} P_{p} \zeta_{k}\left|h_{1}\right|^{2}}
\end{aligned}
$$

From the derived expressions in (30), (31) and (32), when the users have same path-loss we can obtain $a_{k}=a_{1}$. At high SNR values (30), (31) and (32) can be reduced to

$$
a_{k}=\frac{\zeta_{1} a_{1}}{\zeta_{k}}
$$

Substituting (33) into (23), we can get

$$
a_{1}=\frac{1}{\zeta_{1} \sum_{k=1}^{K} \frac{1}{\zeta_{k}}}
$$

Finally, substituting (34) into (33) we obtain

$$
a_{k}=\frac{\zeta_{1} a_{1}}{\zeta_{k}}=\frac{1}{\zeta_{k} \sum_{k=1}^{K} \frac{1}{\zeta_{k}}}
$$

If the users have the same path-loss, i.e., $\zeta_{1}=. .=\zeta_{k}=$ ..$\zeta_{K}$, (35) becomes $a_{k}=\frac{1}{K}$. This explains that, in uniform path-loss model the Min-Sum power allocation scheme reduces to EPA.

\section{Numerical Results}

In this section we present some numerical and simulation results of the derived expressions. Monte-Carlo simulations are performed with $10^{6}$ independent trials. For simplicity, equal noise variances are assumed at the users, $\sigma^{2}$, thus the transmit SNR $\left(\eta_{t}\right)$ is defined as $\eta_{t}=\frac{P_{p}}{\sigma^{2}}$, and the path-loss exponent is assumed to be $m=2.7$.

In Fig. 1, we show the exact and approximate average SEPs with respect to the transmit SNR, $\eta_{t}$, for various input types, BPSK, QPSK and 8-PSK. Fig. 1a, presents the SEPs when $N=K=3$, and Fig. 1b, presents the SEPs when $N=5$, and $K=3$. For seek of comparison, some simulation results of the ZF scheme are also presented in these figures. Firstly, it is clear from these results that the approximate and exact results are in well agreement. It is also apparent that the SEPs degrades with increasing the transmit SNR, $\eta_{t}$, and CI precoding technique always has better performance than $\mathrm{ZF}$ technique with an up to $14 \mathrm{~dB}$ gain in $\eta_{t}$ for a given SEP. In addition, from the results in Figs. 1a and $1 \mathrm{~b}$ we can observe that increasing number of the antennas at the BS reduces 


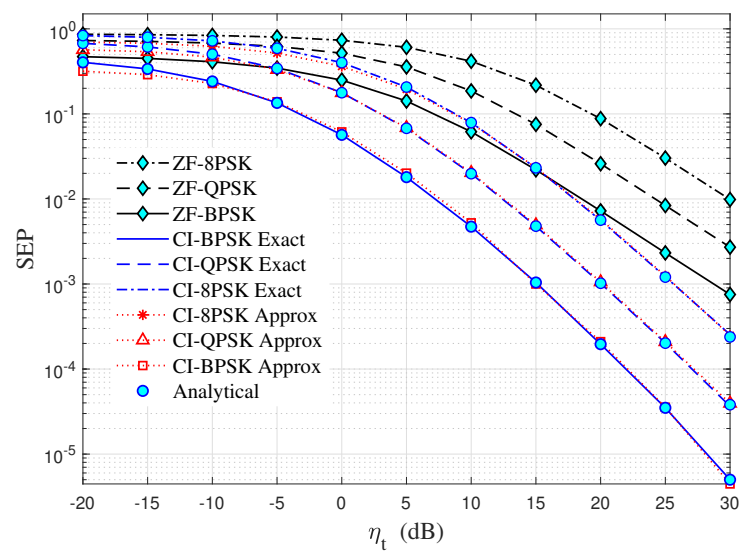

(a) SEP versus transmit SNR, $\eta_{t}$, for BPSK, QPSK and 8-PSK, when $N=K=3$.

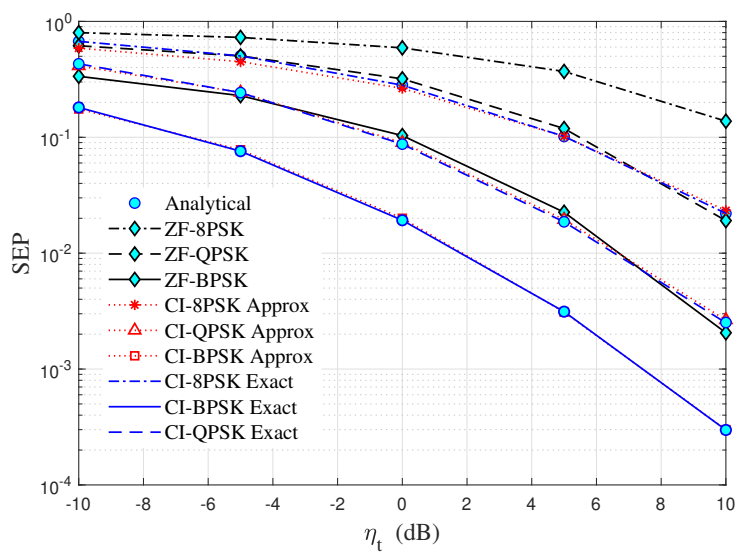

(b) SEP versus transmit SNR, $\eta_{t}$, for BPSK, QPSK and 8-PSK, when $N=5, K=3$.

Figure 1: SEP versus transmit SNR for different input types, when $N=3,5$ and $K=3$.

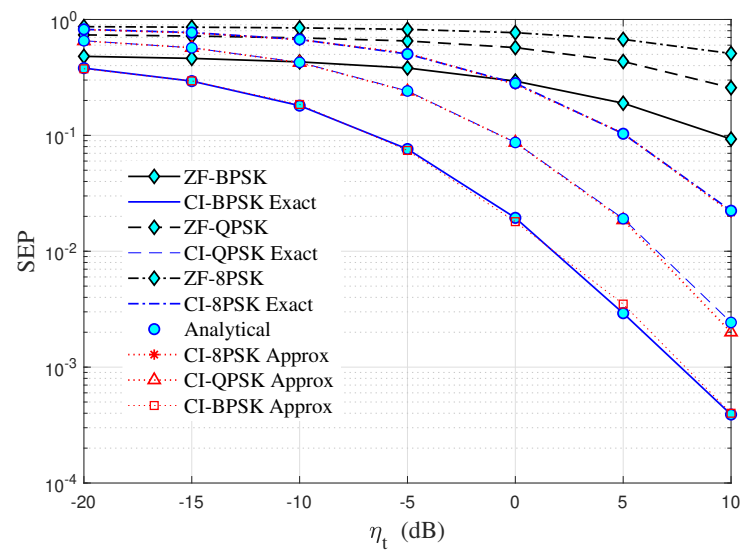

(a) SEP versus transmit SNR, $\eta_{t}$, for BPSK, QPSK and 8-PSK, when $N=K=5$.

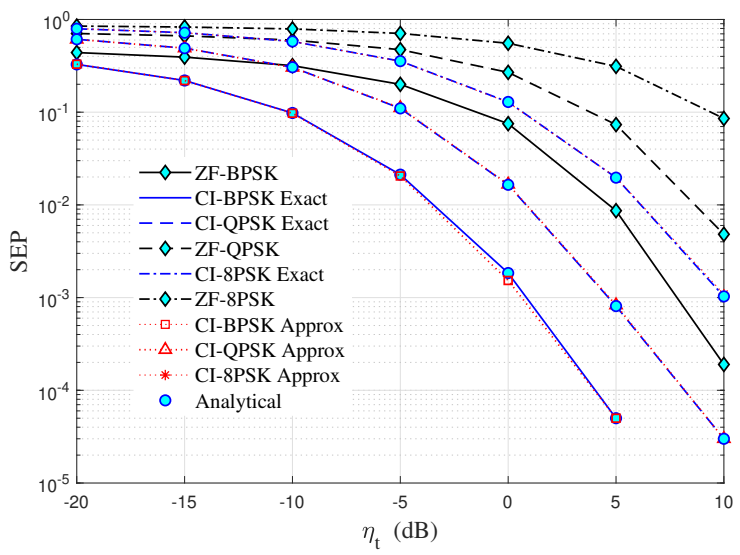

(b) SEP versus transmit SNR, $\eta_{t}$, for BPSK, QPSK and 8-PSK, when $N=10, K=5$.

Figure 2: SEP versus transmit SNR for different input types, when $N=5,10$ and $K=5$.

the SEPs, and the gap between the CI and ZF precoding techniques.

To illustrate the effect of users number on the average SEP, in Fig. 2 we present the average SEPs for the CI and ZF precoding techniques, when $N=K=5$, as in Fig. 2a and when $N=10, K=5$ as in Fig. 2b. From Figs. 1 and 2, it is evident that increasing number of users and antennas result in enhancing the system performance. Furthermore, the CI precoding provides additional up to $16 \mathrm{~dB}$ gain in $\eta_{t}$ compared to $\mathrm{ZF}$ scheme.

Fig. 3 depicts the average SEP as a function of the transmit SNR, $\eta_{t}$, for EPA, and Min-Sum power allocation schemes. Fig. 3a, shows the SEP when $N=K=3$, and Fig. 3b, shows the SEP when $N=K=8$. From these results it is clearly visible that, EPA scheme achieves higher SEP than Min-Sum scheme. Furthermore, by comparing the results in Figs. $3 \mathrm{a}$ and $3 \mathrm{~b}$ we can clearly notice that, the superiority of Min-Sum scheme is more obvious when number of users and BS antennas are large.

\section{CONCLUSIONS}

In this paper the performance of CI precoding technique has been investigated. Firstly, new exact closed-form expression of the MGF was derived. Based on this, the average SEP of CI precoding has been calculated. In this regard, exact analytical expression of the SEP for CI precoding with $M$-PSK was obtained. Additionally, accurate asymptotic expression for the average SEP has been presented. Then, building on the new expressions of the SEP, a power allocation scheme to minimize the total SEP was studied and closed form expression of the power allocation factors were obtained. The numerical results explained that, the CI scheme has much lower SEP than ZF scheme in the all considered scenarios. Furthermore, MinSum power allocation technique can perform much lower SEP compared to EPA scheme.

\section{REFERENCES}

[1] C. B. P. Howard Huang and S. Venkatesan, MIMO Communication for cellular Networks. Springer, 2012, 2008. 


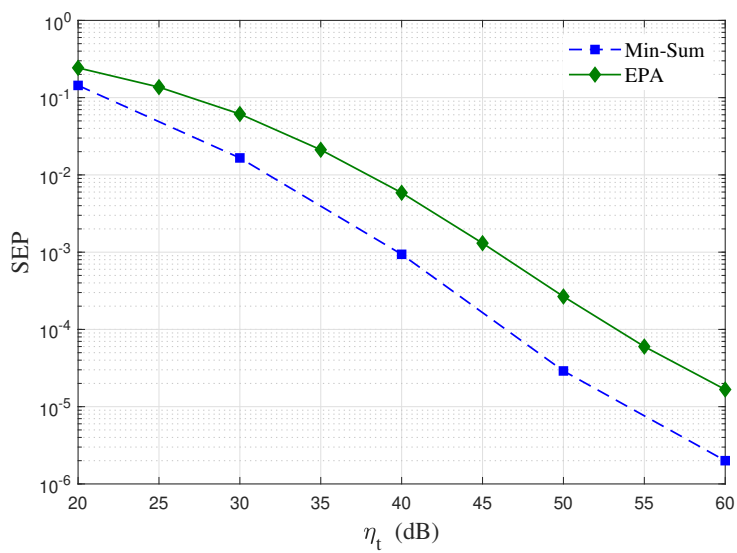

(a) SEP versus transmit SNR, $\eta_{t}$, with different power allocation schemes and QPSK input, when $N=K=3$.

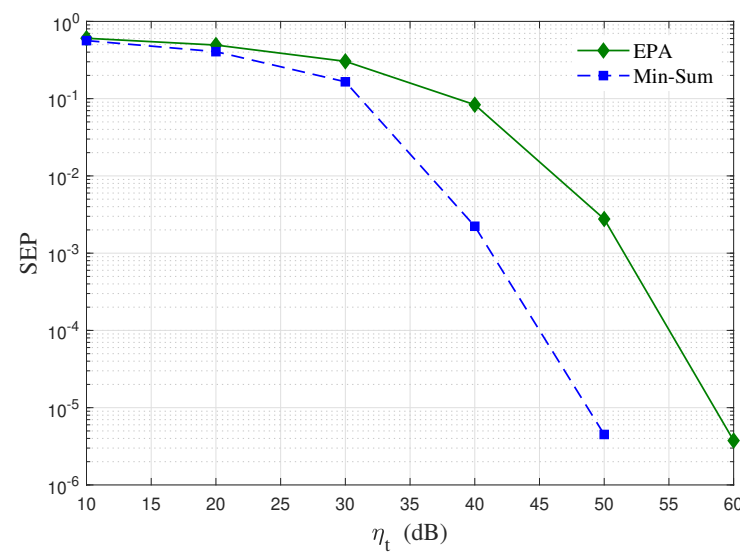

(b) SEP versus transmit SNR, $\eta_{t}$, with different power allocation schemes and QPSK input, when $N=K=8$.

Figure 3: SEP versus transmit SNR with different power allocation schemes.

[2] Y. Wu, C. Xiao, X. Gao, J. D. Matyjas, and Z. Ding, "Linear precoder design for mimo interference channels with finite-alphabet signaling," IEEE Transactions on Communications, vol. 61, no. 9, pp. 3766-3780, September 2013.

[3] A. Salem and K. A. Hamdi, "Wireless power transfer in multi-pair two-way af relaying networks," IEEE Transactions on Communications, vol. 64, no. 11, pp. 4578-4591, Nov 2016.

[4] M. Costa, "Writing on dirty paper (corresp.)," IEEE Transactions on Information Theory, vol. 29, no. 3, pp. 439-441, May 1983.

[5] T. Haustein, C. von Helmolt, E. Jorswieck, V. Jungnickel, and V. Pohl, "Performance of mimo systems with channel inversion," in Vehicular Technology Conference. IEEE 55th Vehicular Technology Conference. VTC Spring 2002 (Cat. No.02CH37367), vol. 1, May 2002, pp. 35-39 vol.1.

[6] C. B. Peel, B. M. Hochwald, and A. L. Swindlehurst, "A vector-perturbation technique for near-capacity multiantenna multiuser communication-part i: channel inversion and regularization," IEEE Transactions on Communications, vol. 53, no. 1, pp. 195-202, Jan 2005

[7] A. Li and C. Masouros, "Interference exploitation precoding made practical: Optimal closed-form solutions for psk modulations," IEEE Transactions on Wireless Communications, pp. 1-1, 2018.

[8] C. Masouros and G. Zheng, "Exploiting known interference as green signal power for downlink beamforming optimization," IEEE Transactions on Signal Processing, vol. 63, no. 14, pp. 3628-3640, July 2015.

[9] C. Masouros and E. Alsusa, "Dynamic linear precoding for the exploitation of known interference in mimo broadcast systems," IEEE Transactions on Wireless Communications, vol. 8, no. 3, pp. 1396-1404, March 2009.

[10] C. Masouros, M. Sellathurai, and T. Ratnarajah, "Vector perturbation

[16] M. Abramowitz and I. A. Stegun, Handbook of Mathematical Functions With Formulas, Graphs, and Mathematical Tabl, Washington,D.C.: U.S. Dept. Commerce, 1972. based on symbol scaling for limited feedback miso downlinks," IEEE Transactions on Signal Processing, vol. 62, no. 3, pp. 562-571, Feb 2014.

[11] A. Salem, C. Masouros, and K. Wong, "Sum rate and fairness analysis for the mu-mimo downlink under psk signalling: Interference suppression vs exploitation," IEEE Transactions on Communications, pp. 1-1, 2019.

[12] A. Salem, C. Masouros, and B. Clerckx, "Rate Splitting with Finite Constellations: The Benefits of Interference Exploitation vs Suppression," arXiv e-prints, p. arXiv:1907.08457, Jul 2019.

[13] A. Salem and C. Masouros, "Error Probability Analysis and Power Allocation for Interference Exploitation Over Rayleigh Fading Channels," arXiv e-prints, p. arXiv:1910.03102, Oct 2019.

[14] M. R. A. Khandaker, C. Masouros, and K. K. Wong, "Constructive interference based secure precoding: A new dimension in physical layer security," IEEE Transactions on Information Forensics and Security, vol. 13, no. 9, pp. 2256-2268, Sept 2018.

[15] R. J. Muirhead, Aspects of Multivariate Statistical Theory, 1982.

[17] M. K. Simon and M. S. Alouini, Digital Communication over Fading Channels. John wiley and Sons, Inc., 2000.

[18] M. R. Mckay, A. Zanella, I. B. Collings, and M. Chiani, "Error probability and sinr analysis of optimum combining in rician fading," IEEE Transactions on Communications, vol. 57, no. 3, pp. 676-687, March 2009.

[19] M. Chiani, D. Dardari, and M. K. Simon, "New exponential bounds and approximations for the computation of error probability in fading channels," IEEE Transactions on Wireless Communications, vol. 2, no. 4, pp. 840-845, July 2003.

[20] S. Boyd and L. Vandenberghe, Convex Optimization. Cambridge, UK: Cambridge University. Press, 2004. 